Colloque C3, suppl. au Journal de Physique III, Vol. 1, octobre 1991

DYNAMIC RUPTURE OF THIN-WALLED CYLINDRICAL SHELLS

\title{
A. G. IVANOV
}

All-Union Scientific Research Institute of Experimental Physics, 607200 Arzamas, Nizhni Novgorod region. USSR

There are some specific features in the rupture behavior of material under dynamic loading. Generally, the short times in which the loads are applied, make it impossible to identify weaknesses and follow the evolution of mupture. The process is still more complicated because of inertial forces involved therein. This mupture may be illustrated, for example, by that of a momentum-accelerated thin-wall ring of plastic material as it expands radially. A lot of papers have been concermed with the solution of this problem aimed to find the rupture strain (or time) and the spectrum (or characteristic size) of fragments. References on this issue are included in papers $[1,2]$. In recent years, two trends in phenomenological description of this event have distinquished themselves, both based on the energy balance. The papers of the first trend claim, that the work of ring mupture into fragments is done by inertial forces, the alternative trend considering elastic ones.

Some papers by American researchers Grady, Kipp and Hightower $[2,3,4]$ are related with the first trend. Underlying in this set of papers is the one by Grady $[5]$. Ref. [2] proves the equation of motion and shows the continuity of the sclution obteined. Thus, assuring the work of mpture is zero, 
the solution found in [2] will transform to the known Mott's solution [6].

The alternative trend has been developed in the papers by the author and his associates $[7,8,9]$ and also by French researchers Olive and Stelly and their colleaques $[10,11]$. These papers consider elastic forces as dominating.

Let us address ourselves to the analysis of research works on ring ruptures, which have been carried out in terms of inertial force effects.

In ref. [2], the Dugdale-Barenblatt opening crack concept is taken as an approximation mechanism of dymamic mupture of a plastic material. The force acting between the crack edges $\left(T_{f}\right)$ is defined as

$$
T_{f}=T_{y}\left(1-x / x_{0}\right) \quad 0<x \leqslant x_{0}, \quad T_{f}=0 \quad x>x_{0}
$$

$\left(T_{y}\right.$ is tensile force, $x_{0}$ - critical crack opening displacement).

Circumferential extension of a ring when it is moving radially, apparently much resembles that of a bar which ends move in opposite directions at velocity $\mathrm{V}_{0}$. Therefore the original equations and final results were obtained for this bar model and extended to the expanding ring case without any foundation. The mupture was assumed to develop in the middle of the bar $\left(x_{0}=0\right)$. The problem being smmetric, a half of the bar from $x=0$ to $x=I_{0}$ was considered. At $x=0$, force $\mathbb{P}_{\mathrm{y}}-\mathrm{T}_{f}$ is existing and the boundary velocity $v=0$, and at $\mathrm{x}=I_{0}$ there is tensile force $\mathrm{T}_{\mathrm{y}}$ and $\mathrm{v}=\mathrm{v}_{0}$. It was claimed, that as the rupture was developing, there formed a region of clastic material in the neigborhood, which was moving as a woic 
at the velocity of elastic-plastic boundary particles. From [6], the equation of motion for this boundary was found from the equality of the bar momentum and the acting force changes. The resulting solution provided formulations for the rupture time $\left(t_{0}\right)$ and strain $\left(\varepsilon_{f}=\dot{\varepsilon} t_{0}\right.$, where $\left.\dot{\varepsilon}=v_{0} / \ell_{0}\right)$ while the double value of the unloaded region by the time of rupture determined the characteristic length of a fragment $\left(x_{f}\right)$.

$$
\begin{aligned}
& t_{0}=2 T_{y}^{-1}\left(9 \rho \gamma^{2} / \dot{\varepsilon}\right)^{1 / 3} \\
& x_{f}=\left[24 \gamma /\left(\rho \dot{\varepsilon}^{2}\right)\right]^{1 / 3}
\end{aligned}
$$

where $\rho, \gamma$ and $\dot{\varepsilon}$ are weight of the bar unit length, specific work of material tear-off and strain rate, respectively.

It. is of interest to ascertain also what source of energy can drive the bar rupture to fragments. Assume, that a bar of $I_{0}$ length, with its ends moving at velocities 0 and $v_{0}$, is mentally cut into $n$ equal parts (fig.1). Using the law of conservation of momentum, one would clearly see, that this action makes the bar kinetic energy redistribute. There rould be a marked increase in the kinetic energy of the fragments' centers of mass (c.m.) due to sharpfall of that inside the fragments.

Thus, the kinetic energy related to a fragnent c.m. is

$$
\begin{gathered}
A_{n}=2 \cdot \int_{0}^{l_{0} / 2 n} 0,5 \rho v^{2} d x=M v_{0}^{2} /\left(24 n^{3}\right), \\
\quad\left(v=v_{0} x / l_{0}, M=\rho l_{0}\right)
\end{gathered}
$$


while in continuous bar $(n=1)$ this is $A_{1}=\mathrm{Nv}_{0}^{2} / 24$

The energy which has converted into the c.m. kinetic energy of motion is

$$
\Delta A=A_{1}-n A_{n}=\left(1-n^{-2}\right) A_{1}
$$

When the stored kinetic energy has been found by formula (4), which may be spent on the rupture, n value can be derived from the equality of $E_{n}$ and the work of rupture $\gamma$; With $n$ known, define $x_{f}$ value just as $\ell_{0} / n$.

$$
x_{f}=\left[24 \gamma /\left(\rho \dot{\varepsilon}^{2}\right)\right]^{1 / 3}
$$

The If $_{f}$ value found agrees with formula (4) (This also agrees with both (6) in [4] and (12) in [12]). This is no extraodinary, because $x_{f}$ value is only determined by the extension of the region where kinetic energy respective to c.m. of fragments has been taken. By formula (5); $x_{f}$ is independent of both the form and the very quantity of $\mathrm{T}_{f}$. Feither does it depend on elastic characteristics of material, i.e. $T_{y}$ and Young's modulus (i). Therefore, if the bar is of liquid, then $x_{f}$ value would conserve according to (9).

How suitable is it in considering inertial forces to extend the results obtained from the enalysis of unidimensional straight motion, to the motion of cylindrically expanding ring? Clearly, every material point of the ring is involved only in radial motion, its energy being $\mathrm{k}_{1} \mathrm{v}_{0}^{2} / 2$, where $\mathrm{i}_{1}$ is ring mass. Is there also any kinetic energy resulting from the velocity gradient along the circumference? By analocy with bar, if $A_{1}$ is formaily calc dated for eny arbitrary 
point on the ring, then it will be found to be 3.3 times as much as radial motion kinetic energy. Moreover, when the ring is cut into $n$ parts, this would have no effect on its fragment motions, energy value $\Delta A=\left(1-n^{-2}\right) A_{1}$ merely vanishing. There is energy imperfection. The following conclusions can be drawn from the above statements:

8) In considering inertial forces, the results obtained for bar can not be extended to ring.

b) When ring is moving radially, the existing inertial forces are due to only radial motion. Therefore, it would be incorrect to assume that the work of ring fragmentation is done by inertial forces.

Consider the papers of the second trend, which are focused on elastic energy. Thus, in ref. [7], it was assumed without any restrictions posed on ring rupture mechanism, that the work Practure was due to elastic tension energy. It was supposed, that as the ring atarts moving its fracture would begin in some section of this. The required elastic energy for rupture would be taken from the section neighborhood at the unstressing wave velocity. The balance of energy and work of mupture was written as

$$
\int^{V} q d V=\lambda S
$$

where $q$ and $\lambda$ ere the specific (elastic) energy and voris of rupture, and $S$ and $V$ are the ring section and unstressed volume of the section neighborhood. The material was assumed viscoelastoplastịc. 
Straining equation was taken in the form

$$
\sigma=\sigma_{0}+\eta \dot{\varepsilon}
$$

where $\sigma_{j}$ is static flow stress, $\eta$ - viscosity. Combined solution of (6) and (7) yields a function of the form $F\left(\varepsilon_{f}, \dot{\varepsilon}, \alpha, \mu\right)$ which for $\varepsilon_{f} \ll 1$ simplifies to

$$
\begin{aligned}
& \varepsilon_{f}=\dot{\varepsilon} \alpha /(1+\dot{\varepsilon} \mu)^{2} \\
& \left(\alpha=4 E \lambda /\left(3 c \sigma_{0}^{2}\right), \mu=\eta / \sigma_{0}\right) .
\end{aligned}
$$

Function 7 and relation (8) yield maximum value when strength $\left(\sigma_{0}\right)$ and viscous $(\eta \dot{\varepsilon})$ terms are equal in eq. (7). This approach was successfully extended in refs. $[11,10]$ to describe the ruptures of radially expanding spheric shells. In ref. [8], the expression for fragments number was found for $\varepsilon_{f} \ll 1 \quad n=3 \pi\left(R_{0} \sigma_{0}+v_{0} \eta\right)^{2} \cdot\left(4 R_{0} E \lambda\right)^{-1}$.

A more general equation of straining, than (12), was used in ref. $[8]$. There the volume swept by unstressing was determined in terms of circumferential material displacement with $R$ incre. se. Moreover, it was observed, that for liquids $\sigma_{0}=0$ and elastic energy results from only viscous forces $(\eta \dot{\varepsilon})$. Therefore, $\varepsilon_{f}(\dot{\varepsilon})$ should not be a rising function.

Refer to an experiment, as mentioned above, both approache under consideration are based on the balence of energy needed for mupture and work of forces (inertial or elastic) capable of this. The results of experimental studies are given in refs. $[2-4],[7-11]$, winch are claimed by the authors to support both of the approaches. However, the available experimental data are insufficient to bring the issue to a close, since there are too meny simplifying assumptions in the above-menti- 
oned papers (such as $\mathrm{T}_{\mathrm{y}}=$ const, $\gamma($ or $\lambda)=$ const, $\eta=$ const etc.). Yet now there are good reasons to favour the approach based on elastic forces. These are as follows

1. For viscoelastoplastic materials, this solution predicts the occurrence of plasticity peak in ring or stripe under biaxial strain [13]. This peak was observed experimentally for soft steels in papers $[7,8]$, its occurrence for steels and aluminum-titinium alloys being pointed out in book [14]. It is noted in $[11]$, that relation $\varepsilon_{f}(\dot{\varepsilon})$ having a maximum may account for the falling sections of function $\varepsilon_{f}(\dot{\varepsilon})$. for a number of alloys. To illustrate this, figure 2 , as taken from $[14]$, shows $\varepsilon_{\xi}(\dot{\varepsilon})$ relations.

2. As shown above, when ring is expanding radially, there are no inertial forces directed circumferentially. These may occur only during the rupture of real ring material into fragments due to their elastic unstressing.

Thus, it is elastic strain energy which is considered a driving factor in the dynamic mupture of material in its deeply-plastic region, as well as in the static fracture. 
1. Odintsov V.A., Chudov I.A. In: Mekhanika, N 5, Problemy dinamiki uprugoplasticheckikh sred, M., "Mir", 1975, p.p. $85-159$.

2. Kipp M.E. and Grady D.E.J.Mech.Phys.Solids. 1985, v.33, N 4, p.p:399-415.

3. Grady D.E. and Kipp M.E. Mech. of Mat.1985, v.4, p.p. 311-32,

4. Grady D.E. and Hightower M.M. Int.Conf. on Shock-Wave and High-Strain-Rate Phen. in Mat.San-Diego, USA, August 12-17, 1990.

5. Grady D.E,J.Appl.Phys. 1982, v.53,.N 1, p.322-325.

6. Mott N.F. Proc.R.Soc.A, 19.47, v.189,p.300.

7. Ivanov A.G. Probl.prochn., 1976, H 11,p.50.

8. Ivanov A.G., Kochkin I.I. et al., PMPF, 1983, if 1, p.p. 112-117.

9. Ivanov A.G. PMPF, 1986, N 2, p.p.146-151.

10.0live F., Nicand A. et al.

"Rupture Behavior of Hetals in Explosive Expansion in Wech. Prop.High Rates Strain". Bristol - Iondon, 1980, $p \cdot p \cdot 242-251$.

11. Stelly M., Legrand J. and. Dormeval R. "Some lietallurgical. Aspects of the Dymamic Expansion of Shells in Shock Haves and High Strain Rate Phenomena in Hetals". Plenum Press, Hew York, 1981, p.p.113-125.

12. Grady D.E. and Kipp. Li.E.. J.IIech. Phys.Solids.1987, v. 35 , II 1 , p.p. 95-119.

13. Serikov S.V. PliTF, 1982, if 6, p.p.p123-129.

14. Borisevich V.K., Sabel'kin V.P. et al. In: sb.trudov Kharkovskogo Aviutsionnot 2 Instituta. Imp.obrab.metallov dav1., Kharkov, 1981, II 9, p.p.75-82. 


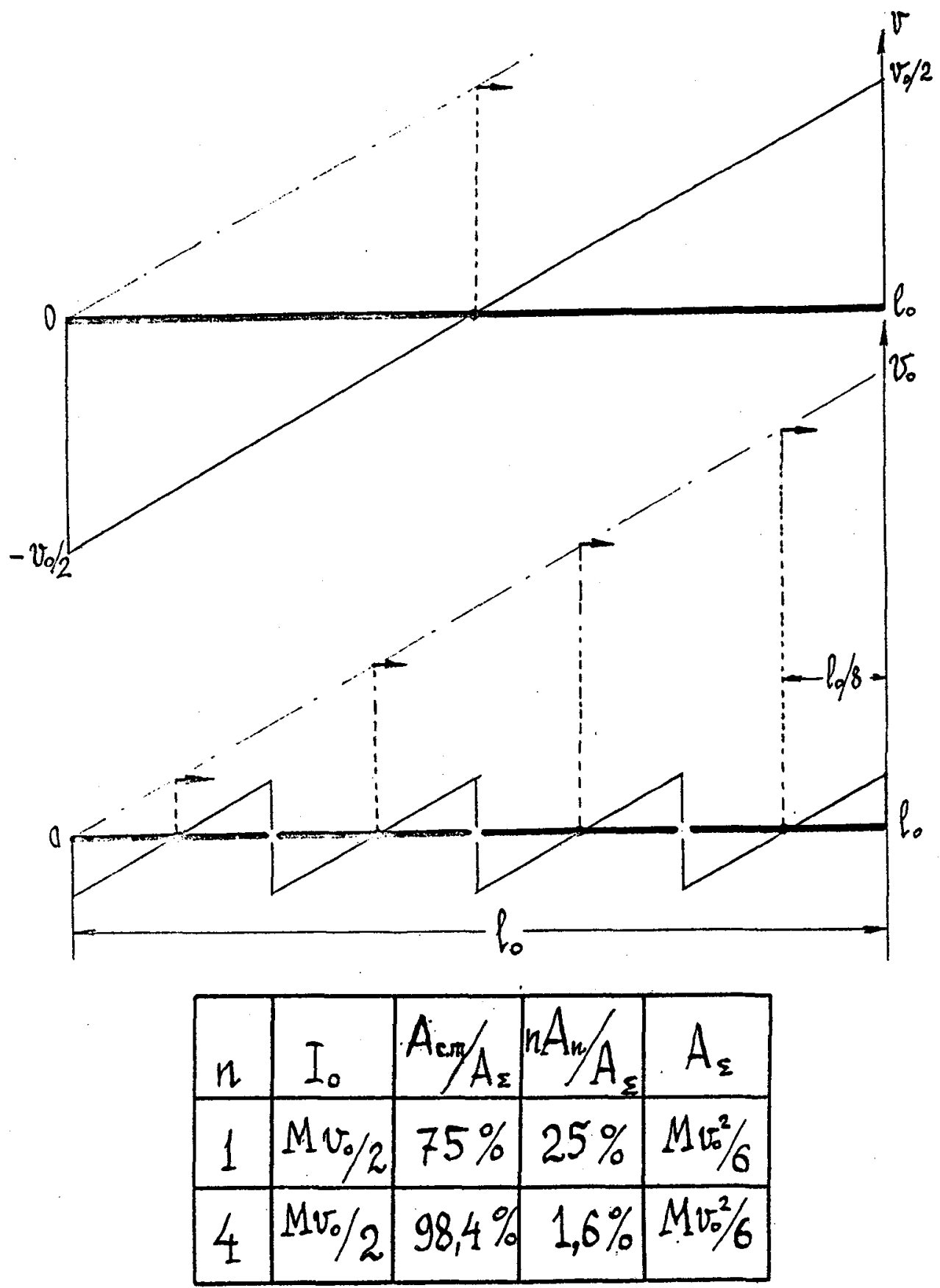

Fig.1. Schematic representation of kinetic energy redistribution when bar is cut into $n$ parts $(n=4)$. 


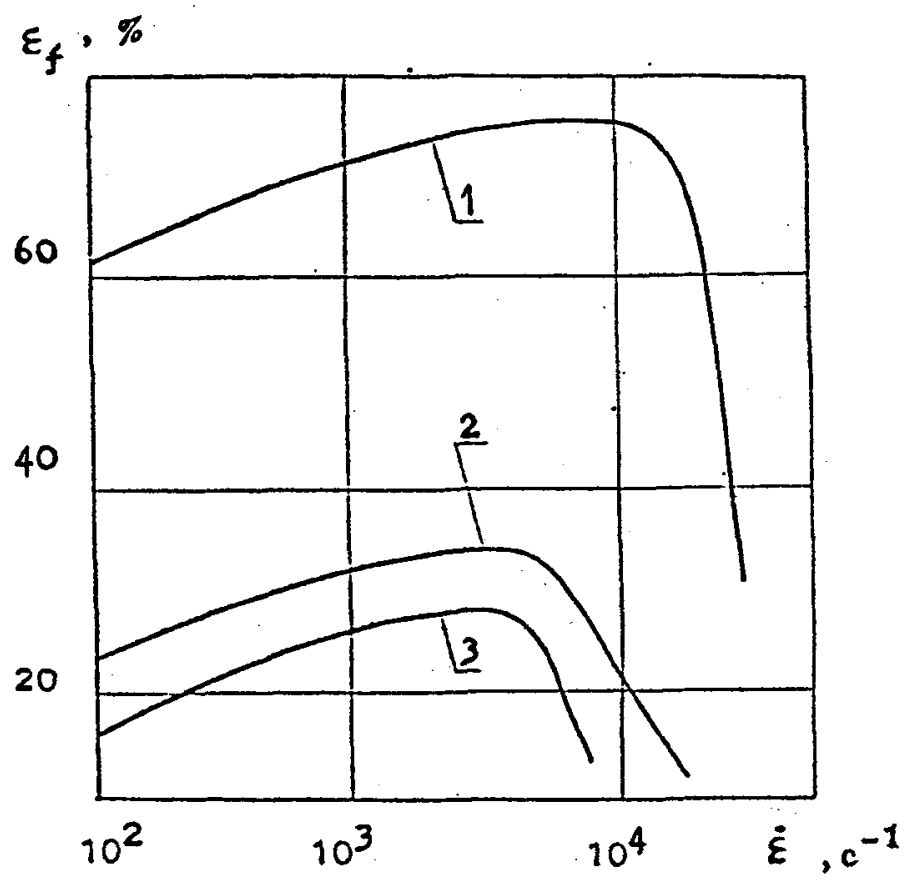

Fig.2. Unit elongation as a function of strain rate.

1 - steel IXI8H9T, 2 - annealed steel 40,

3 - annealed alloy $\mathrm{D}-16$. 